\title{
Molecular-dynamics study of mechanical properties of copper
}

\author{
P. HEINO ${ }^{1}, \mathrm{H}$. HÄKKINEN ${ }^{2}$, and K. KASKI ${ }^{1}$ \\ ${ }^{1}$ Helsinki University of Technology, Laboratory of Computational Engineering \\ Miestentie 3, P.O. Box 9400, FIN-02015 Espoo, Finland \\ ${ }^{2}$ University of Jyväskylä, Department of Physics \\ P.O. Box 35, FIN-40351 Jyväskylä, Finland
}

(received 21 July 1997; accepted in final form 2 December 1997)

PACS. 34.20Cf - Interatomic potentials and forces.

PACS. 62.20-x - Mechanical properties of solids.

PACS. 81.05Bx - Metals, semimetals, and alloys.

\begin{abstract}
Mechanical properties of copper have been studied using effective-medium theory and Molecular-Dynamics simulations. At room temperature we calculate the tensile moduli of systems that are elongated along different crystal orientations. These moduli are in very good agreement with the experimental values, the difference being less than $6 \%$. The elastic constants obtained from simulations were also in good agreement with experiments. In addition, the point of maximum stress is found to be of the same order of magnitude as the experimental value. Also crack propagation in systems with periodic boundaries has been studied and micro-voids are seen to generate near the crack tip. Crack propagation is found to be a result of coalescing micro-voids.
\end{abstract}

Introduction. - Recently, the dynamics of fracture has received considerable interest. From the physics point of view fracture in solid materials involves processes on a wide range of length and time scales. Theoretically different length scales can be treated with macroscopic Finite-Element models [1], mesoscopic spring models [2] or by using truly microscopic approach with interatomic potential. Because in the atomistic case a realistic potential can be hard to find, approximate pair potentials are often used. For example Morse [3], [4], Lennard-Jones (LJ) [5], [6] or Johnson [3], [7] potentials are quite common. However, when the Morse and LJ potentials were compared with a more realistic many-body potential [5], pair potentials were found to yield brittle behaviour, while ductile materials must be described with a many-body potential. On the other hand, in dynamic fracture simulations large system sizes are necessary, which is why so far simulations have mostly been done by using a simple pair potential in a two-dimensional system [6], [7] or in a system with otherwise less degrees of freedom [8]. Till recently simulations in $3 \mathrm{D}$ with a realistic many-body potential for describing the behaviour of ductile materials, e.g. copper, could be realized only in small systems [9]. However, with the rapid development of parallel processing large-scale multi-million atom simulations with many-body potentials have become feasible [10].

Also in this work we study the elastic properties of copper using a realistic many-atom but now ab initio type potential obtained from the effective-medium theory. Unlike the simulations by Zhou et al. [10] — done practically at zero temperature - we have studied the system at room temperature. One of our goals is to study the goodness of the many-atom potential in giving reliable results for elastic constants in comparison with the experimentally measured values and how they depend on the system size. In addition, we study the critical stress and strain of the system for failure condition. We will also study the propagation and growth of an initial crack under tensile mode I loading condition along various fcc crystal orientations.

(c) EDP Sciences 
(a)

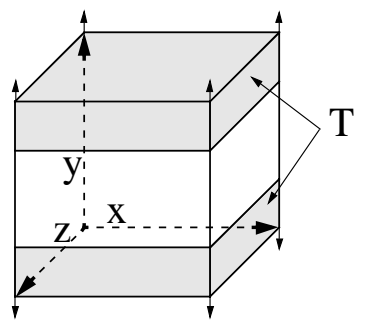

(b)

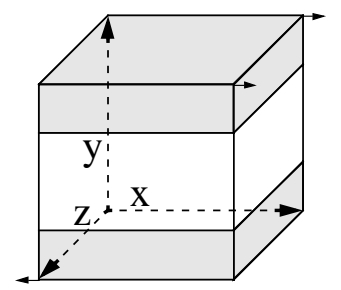

Fig. 1. - Tensile (a) and shear (b) test setups, where atoms on the fixed boundaries (topmost, maximum $y$ and bottommost, minimum $y$ ) are displaced with time-dependent rate in 3 different crystal orientations (cf. text). Atoms in the shaded areas are in contact with the thermostat throughout the test.

Potential model. - In our model the interactions between copper atoms are described by the effective-medium theory (EMT) [11], which is an approximate method for calculating the total energy of an arbitrary arrangement of metal atoms from their spatial positions. EMT has been shown to be a powerful scheme for describing various bulk and surface properties of metals [12]-[18]. The many-atom nature of metallic cohesion is crucial in most of these problems, which is the point where EMT has a clear advantage over the classical pair potentials. Furthermore, EMT provides a useful scheme to understand the metallic cohesion based on $a b$ initio calculations using the jellium model and density-functional theory with the local-density approximation [19]. The main contribution to cohesion comes from the embedding energy of an atom when it feels the background electron density at its lattice site. There are two corrections: one arises from the one-electron energy spectrum and the other from the imperfections of the lattice (so-called atomic sphere correction). While the first correction is important only for clusters or for some processes at surfaces [18] and therefore not included here, the latter is important to our study since it largely defines the elastic properties of the system. In this work we extend the atomic interactions over three nearest neighbours of the fcc lattice [12]. This improves molecular-dynamics results for several thermodynamic quantities and defect energies [12], [14]. For details of the potential see [12].

Simulation method. - With the above potential model we simulated both tensile and shear experiments (fig. 1) using the Molecular Dynamics (MD) [20] method. In MD the Newtonian equations of motion are numerically solved using the velocity Verlet algorithm as the time integrator [20]. Initially the atoms were placed to the sites of the fcc lattice by adding small Gaussian random deviation to the coordinates and their velocities were selected from the finite-temperature $(T=293 \mathrm{~K})$ Maxwell distribution. The system was thermalized with a Nosé-Hoover chain (NHC) $[21]$ thermostat of length $(M=50)$ for approximately 6 ps $(\approx 700$ time steps).

In the tensile test the fixed boundaries were moved apart vertically and in shear test horizontally (fig. 1). These boundary atoms were displaced from their initial positions using the time-dependent strain rate $\dot{\epsilon} \sigma\left(t, t_{0}, \alpha\right)$. Here $\dot{\epsilon}=113 \% / \mathrm{ns}$ is the maximum strain rate and $\sigma\left(t, t_{0}, \alpha\right)$ is a sigmoid-function (smooth step) with $\sigma\left(t, t_{0}, \alpha\right)=\left(1+e^{\alpha\left(t-t_{0}\right)}\right)^{-1}$, where $t$ is time and $\alpha$ determines the maximum acceleration. These parameters were selected such that $t_{0}$ was the time in which an elastic wave from the fixed boundary can propagate back and forth the system, and $\alpha<0$ was selected such that the boundaries reached $99 \%$ of the maximum velocity in $2 t_{0}$.

During test only half of the system (atoms near fixed boundaries, see fig. 1) was in contact with the NHC [21] thermostat $(M=50)$ at room temperature to mimic heat transfer from the system to the load. This is because we wanted to minimize the possible disturbance of 
the thermostat on crack dynamics. Three different crystal orientations were studied with orthogonal $x, y, z$ directions being $([100],[010],[001]),([01 \overline{1}],[011],[100])$ or $([\overline{1} \overline{1} 2],[111],[1 \overline{1} 0])$.

Elastic constants. - From the simulation (fig. 1) we determined the tensile and shear modulus of the system. At room temperature the dependence of these moduli on the system size was studied in the range of $10^{4}-\left(2.8 \times 10^{5}\right)$ atoms. The modulus was obtained for small values of strain (i.e. $Y_{d, m}=\left.\frac{\partial \sigma}{\partial \epsilon}\right|_{\epsilon=0}$ ) from the second-degree polynomial that was fitted to the stress-strain data. Here the stress is the force needed for the deformation divided by the area it acts on. The subsripts $d, m$ denote the crystal orientation of straining, $d$, and the mode of the test, $m=\|$ for tensile test and $m=\perp$ for shear test. For relatively large systems $\left(>10^{5}\right.$ atoms $\approx 106 \AA$ ) the moduli turned out to be independent of the system size (fig. 2).

When the system has periodic boundaries in both $x$ - and $z$-directions, we may assume that straining results in a displacement field $\mathbf{u}(\mathbf{r})=\mathbf{s}_{\mathbf{0}} \epsilon \mathbf{r} \cdot \mathbf{h}_{\mathbf{0}}-\mathbf{u}_{\mathbf{0}}$. Here $\epsilon$ is the amount of tensile/shear strain, $\mathbf{h}_{\mathbf{0}}$ is the unit vector in $y$-direction, $\mathbf{s}_{\mathbf{0}}$ is the unit vector of straining ( $\mathbf{s}_{\mathbf{0}}=\mathbf{h}_{\mathbf{0}}$ in tensile test) and $\mathbf{u}_{\mathbf{0}}$ is a constant defined by the displacement of the bottom boundary. Then the strain field is constant and the elastic energy of the system is given by $W=\frac{1}{2} V Y_{d, m} \epsilon^{2}$, where $V$ is the volume of the system and by definition the modulus $Y$ equals the modulus given in the previous paragraph. For the above displacement field the moduli $Y_{d, m}$ in terms of the elastic constants $c_{i j}$ are [22]

$$
\left\{\begin{array}{l}
Y_{[010], \|}=c_{11}, Y_{[011], \|}=\frac{1}{2} c_{11}+\frac{1}{2} c_{12}+c_{44}, Y_{[111], \|}=\frac{1}{3} c_{11}+\frac{2}{3} c_{12}+\frac{4}{3} c_{44}, \\
Y_{[010], \perp}=c_{44}, Y_{[011], \perp}=\frac{1}{2} c_{11}-\frac{1}{2} c_{12}, \quad Y_{[111], \perp}=\frac{1}{3} c_{11}-\frac{1}{3} c_{12}+\frac{1}{3} c_{44},
\end{array}\right.
$$

From table $\mathrm{I}$ it is seen that the difference in the tensile moduli ranges from 2 to $6 \%$ and thus the agreement is very good. However, in case of the shear moduli the difference is somewhat larger ranging from 3 to $15 \%$. In the fitted moduli the error bars ( $\approx$ two standard deviations) were of the order $1.5 \%$ except for $Y_{[011], \perp}$, where it was $3 \%$.

Due to the fcc structure of the system, eqs. (1) are not independent, and the following relations for the moduli are obtained:

$$
Y_{[111], \|}=\frac{4}{3} Y_{[011], \|}-\frac{1}{3} Y_{[010], \|}, \quad Y_{[111], \perp}=\frac{2}{3} Y_{[011], \perp}+\frac{1}{3} Y_{[010], \perp} .
$$

Let the moduli calculated from eq. (2) be denoted by *. Then within the error bars the values of $Y_{[111], \|} / Y_{[111], \|}{ }^{*}-1$ and $Y_{[111], \perp} / Y_{[111], \perp}{ }^{*}-1$ range between $1.4-6.1 \%$ and $7.5-14 \%$, respectively. Thus the relations in eq. (2) do not hold within the error bars. However, the difference is not large (less than 11\%) as seen from table I. This difference could be due to distortions in the fcc structure as a result of straining and finite temperature. In addition, the

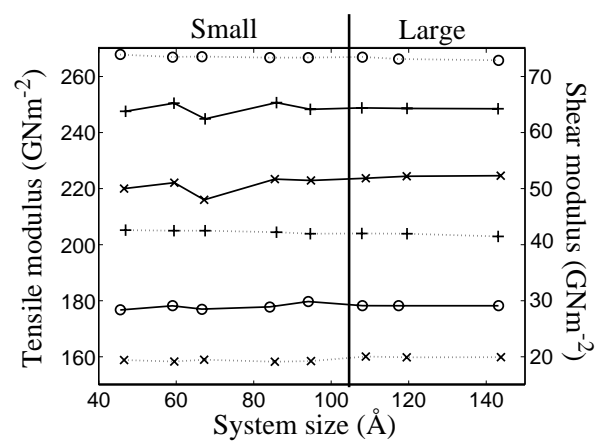

Fig. 2. - Tensile and shear moduli obtained from simulations. The size of the cubic system is indicated by the side length of the cube. The crystal orientation from bottom to top boundary is denoted by $\circ[010], \times[011]$ and $+[111]$. The solid line stands for tensile moduli and the dotted line for shear moduli. 
TABLE I. - Tensile modulus, $Y_{d, \|}$, and shear modulus, $Y_{d, \perp}\left(\mathrm{GNm}^{-2}\right)$ as obtained from simulation tests along different crystal orientations $d$. The experimental moduli were calculated from eq. (2) by using the experimental values for $c_{i j}$. The elastic constants (MD) were calculated with the same equation using the moduli obtained from simulations. For other quantities see text.

\begin{tabular}{lllllll}
\hline & $Y_{[010], \|}$ & $Y_{[011], \|}$ & $Y_{[111], \|}$ & $Y_{[010], \perp}$ & $Y_{[011], \perp}$ & $Y_{[111], \perp}$ \\
\hline Experimental & 168.4 & 220.3 & 237.6 & 75.4 & 23.5 & 40.8 \\
MD & 178.2 & 224.3 & 248.6 & 73.2 & 20.0 & 41.8 \\
& $c_{11}$ & $c_{12}$ & $c_{44}$ & $\frac{Y_{[111], \|}}{Y_{[111], \|}^{*}}-1$ & $\frac{Y_{[111], \perp}}{Y_{[111], \perp}^{*}}$ & $\frac{c_{12}+c_{44}}{c_{11}}$ \\
Experimental & 168.4 & 121.4 & 75.4 & & & 1.169 \\
MD & 176.9 & 132.8 & 73.7 & $3.7 \%$ & $10.8 \%$ & 1.167 \\
\hline
\end{tabular}

structure of copper is not ideal fcc near the boundaries [16], which can be important for small system sizes.

The elastic constants $c_{i j}$ were solved from the simulated moduli by using eqs. (1) and the least-squares method. These results are given in table I, where the difference ranges between $2 \%$ and $10 \%$. It is known that the Cauchy relation $c_{12}=c_{44}$ holds for pair potential models [23], but this has not been observed to be the case for metals. For example in case of copper we get a ratio $c_{12} / c_{44}=1.81$, while the experimental value is 1.61 . However, table I shows that also copper obeys the approximate relation for cubic crystals [11], i.e. $\left(c_{12}+c_{44}\right) / c_{11} \approx 1$. From these comparisons we conclude that EMT describes surprisingly well the elastic properties of copper.

System strength. - When the system is strained much beyond the linear elastic region it fails at some point. However, when some of the boundaries were free, fracture was not observed at that point, only a sharp drop in the stress vs. strain curve occurred. It is well known [24] that in fcc crystals plastic deformation often takes place as slip, and the resulting slip plane is a $\{111\}$-plane. In the simulations with $y=[011]$ and $z=[100]$ this process was seen in the system with free boundaries. Also the resulting strength was very small (cf. table II). The same slip planes were also seen for $y=[010]$. However, when the boundaries of the system were set periodic, slips could not occur in tensile loading tests. Therefore it is understandable that such systems appear strongest.

Brenner [25] has studied the strength (maximum stress) of copper whiskers - which are very small, chemically very pure and almost dislocation-free pieces of copper- to find the dependence of strength on the system size. In his study different size whiskers were elongated in the [111] crystal orientation and the strength was found to increase with decreasing system size and to decrease with increasing disorder. In large systems, however, the strength also depends strongly on defects in the bulk and on free surfaces [26]. Brenner found the strength of the smallest system to be $2.9 \mathrm{G} \mathrm{Nm}^{-2}$, while our simulations gave a value of about $10 \mathrm{GNm}^{-2}$ in the same crystal orientation (table II) but in an extremely small and completely dislocation-free system. In addition, from table II it is evident that boundary effects are very significant. In his studies, Doyama [9] applied tensile strain in the [001] orientation to a considerably smaller system, 4338 atoms, with free boundaries and embedded atom potential, and found a failure strain of $10 \%$, which is close to our $8.5 \%$. From all these it seems that EMT describes well the strength of the system without defects.

Crack propagation. - In the simulations of the system with free boundaries crack propagation was not seen. However, when a system with periodic boundaries failed, crack initiation 
TABLE II. - Maximum tensile stress $\left(\sigma_{\mathrm{f}}, \mathrm{GNm}^{-2}\right)$ and strain $\left(\epsilon_{\mathrm{f}}, \%\right)$ along different crystal orientations and with various boundary conditions. The size of the system is $(70 \AA)^{3} \approx 3 \times 10^{4}$ atoms.

\begin{tabular}{llrlccc}
\hline & \multicolumn{2}{c}{ Free $x$ and $z$} & \multicolumn{2}{c}{ Free $x$, periodic $z$} & \multicolumn{2}{c}{ Periodic $x$ and $z$} \\
\hline & $\sigma_{\mathrm{f}}$ & $\epsilon_{\mathrm{f}}$ & $\sigma_{\mathrm{f}}$ & $\epsilon_{\mathrm{f}}$ & $\sigma_{\mathrm{f}}$ & $\epsilon_{\mathrm{f}}$ \\
{$[010]$} & 6.3 & 8.5 & 6.6 & 8.0 & 15.1 & 15.5 \\
{$[011]$} & 4.4 & 4.5 & 11.7 & 9.5 & 14.3 & 12.0 \\
{$[111]$} & 9.8 & 6.5 & 11.2 & 7.0 & 15.6 & 10.5 \\
\hline
\end{tabular}

was seen, because plastic Poisson-type contraction cannot occur. When a crack initiated, a free surface (that of the crack) was generated into the system. As the energy of the system depends on its electron density and the initial density is energetically favourable, the crack becomes larger, thus resulting in crack propagation.

In order to study how such a mode I crack propagates, we made a small initial void to the centre of the system to serve as a crack seed. With the crystal orientations given above, the system was elongated in the $y$-direction while in the other directions periodic boundaries were imposed. The crack seed $(4 \times 4$ atoms wide tube in the periodic $z$-direction) was introduced to the centre of the system. When the system was elongated in [010] orientation, the crack grew such that its speed in the $x$-direction was roughly twice the speed in the $y$-direction. Later,

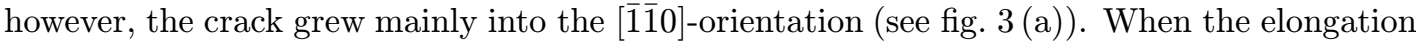
was in [011] orientation, the crack propagated fairly easily in the $x$-([01] $])$-direction. However, in the $z$-([100])-direction the crack propagation was observed much later. Thus the crack formed a tube to the system in the $x$-direction, but the rest of the system forming a "neck" in that direction could still carry load, see fig. 3 (b). There it was also seen that crack propagation in this case was a result of micro-void coalescence. In both cases the crack seemed to favour the $\langle 110\rangle$ crystal orientation.

(a)
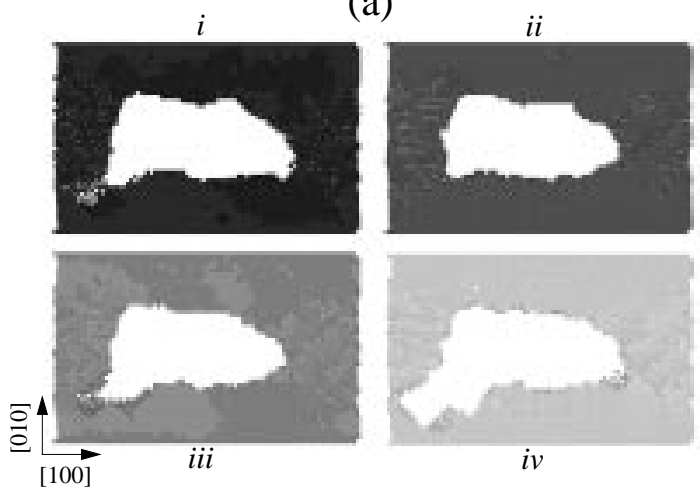
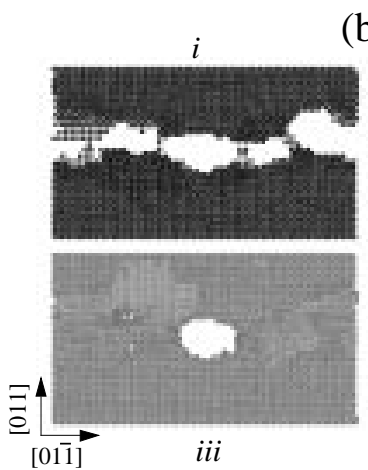

(b)

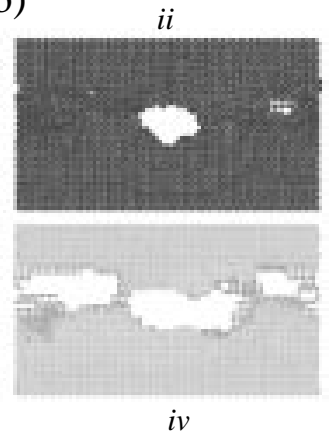

Fig. 3. - System with initial crack seed elongated in the vertical $y$-direction, being [010] in (a) and $[011]$ in (b), while the respective horizontal $x$-directions are [100] and [011] . The $z$-coordinate is shown as shading of the atoms. The total strain is $25.4 \%$ in (a) and $16.6 \%$ in (b). The system is divided into four layers in the $z$-direction such that $(i)$ is furthest away and $(i v)$ nearest. Periodic boundaries are used in both the $x$ - and $z$-direction. The system consists of 15 atomic layers in the $z$-direction and the number of atoms is $\approx 2 \times 10^{4}$. Crack propagation in the $\langle 110\rangle$ orientation is seen in both systems. 
Summary. - Mechanical properties of copper were studied using effective-medium theory and Molecular Dynamics method. At room temperature the elastic constants were found to be in agreement with experimental values. The strength in terms of maximum tensile stress of the system was also found to be in agreement with experimental findings. In the study of crack propagation micro-voids were seen to generate near the crack tip and the crack propagation was a result of coalescing micro-voids. In addition the crack seemed to favour the $\langle 110\rangle$ crystal orientation, in which it grew faster than in other orientations.

Based on this high level of agreement with experimental data we conclude that EMT molecular-dynamics simulations can serve as reliable and realistic tool for studying such complicated mechanical processes in metals as fracture in a sample containing grain boundaries, voids or impurities. In future studies these issues will be addressed.

\section{REFERENCES}

[1] Cox S. J. D. and Paterson L., Phys. Rev. B, 40 (1989) 4690.

[2] Mori Y., Kaneko K. and Wadati M., J. Phys. Soc. Jpn., 50 (1990) 1591; Taguchi Y.-H., Mater. Sci. Eng. A, 176 (1994) 295; Rautiainen T. T., Alava M. J. and Kaski K., Phys. Rev. E, $\mathbf{5 1}$ (1995) R2727.

[3] DeCelis B., Argon A. S. and Yip S., J. Appl. Phys., 54 (1983) 4864.

[4] Baiguzin E. Y., Melker A. I. and Mikhailin A. I., Phys. Status Solidi A, 108 (1988) 205; Zhou S. J., Lomdahl P. S., Thomson R. and Holian B. L., Phys. Rev. Lett., 76 (1996) 2318.

[5] Holian B. L., Voter A. F., Wagner N. J., Ravelo R. J., Chen S. P., Hoover W. G., Hoover C. G., Hammerberg J. E. and Dontje T. D., Phys. Rev. A, 43 (1991) 2655; Wagner N. J., Holian B. L. and Voter A. V., Phys. Rev. A, 45 (1992) 8457.

[6] Abraham F. F., Brodbeck D., Rafey R. A. and Rudge W. E., Phys. Rev. Lett., 73 (1994) 272; Abraham F. F., Phys. Rev. Lett., 77 (1996) 869.

[7] Sieradzki K., Dienes G. J., Paskin A. and Massoumzadeh B., Acta Metall., 36 (1988) 651.

[8] Marder M. and Liu X., Phys. Rev. Lett., 71 (1993) 2471.

[9] Doyama M., Nucl. Instrum. Methods Phys. Res. B, 102 (1995) 107.

[10] Zhou S. J., Beazley D. M., Lomdahl P. S. and Holian B. L., Phys. Rev. Lett., 78 (1997) 479.

[11] Jacobsen K. W., Nørskov J. K. and Puska M. J., Phys. Rev. B, 35 (1987) 7423; JacobSEN K. W., Comm. Condens. Matter Phys., 14 (1988) 129.

[12] Häkninen H. and Manninen M., J. Phys. Condens. Matter, 1 (1989) 9765.

[13] Stoltze P., Jacobsen K. W. and Nørskov J. K., Phys. Rev. B, 36 (1987) 5035.

[14] Häkkinen H. and Manninen M., Phys. Scr., T33 (1990) 210; Häkkinen H., Mäkinen S. and Manninen M., Phys. Rev. B, 41 (1990) 12441.

[15] Jacobsen K. W. and Nørskov J. K., Phys. Rev. Lett., 60 (1988) 2496; Stoltze P., Nørskov J. K. and Landman U., Phys. Rev. Lett., 61 (1988) 440; Surf. Sci., 220 (1989) L693.

[16] Häkkinen H. and Manninen M., Phys. Rev. B, 46 (1992) 1725.

[17] Häkkinen H., Merikoski J., Manninen M., Timonen J. and Kaski K., Phys. Rev. Lett., 70 (1993) 2451; Phys. Rev. B, 49 (1994) 4938; Christensen O. B., Stoltze P., Jacobsen K. W. and Nørskov J. K., Phys. Rev. B, 41 (1990) 12413.

[18] Christensen O. B., Jacobsen K. W., Nørskov J. K. and M. Manninen, Phys. Rev. Lett., 66 (1991) 2219.

[19] Puska M. J., Nieminen R. M. and Manninen M. J., Phys. Rev. B, 24 (1981) 3037.

[20] Allen M. P. and Tildesley D. J., Computer Simulation of Liquids (Oxford University Press) 1987.

[21] Martyna G. J., Klein M. L. and Tuckerman M., J. Chem. Phys., 97 (1992) 2635.

[22] Huntington H. B., The Elastic Constants of Crystals (Academic Press) 1958.

[23] Ercolessi F., Parrinello M. and Tosatti E., Philos. Mag. A, 58 (1988) 213.

[24] Kittel C., Introduction to Solid State Physics, 3rd edition (John Wiley \& Sons) 1967.

[25] Brenner S. S., J. Appl. Phys., 27 (1956) 1484.

[26] Kelly A., Strong Solids, 2nd edition (Oxford University Press) 1973. 